\title{
ERRATUM
}

\section{Analytical theory of homogeneous mean shear turbulence - ERRATUM}

\author{
Jerome Weinstock \\ doi:10.1017/jfm.2014.13, Published by Cambridge University Press, \\ 20 June 2013
}

It has been brought to our attention that two articles published in Journal of Fluid Mechanics were assigned the same Digital Object Identifier (DOI). As a result of this, if readers attempted to use the DOI to resolve to the article, they would have reached only one (Guardone et al. 2014). We have corrected this error by assigning a new unique DOI to Weinstock (2013), namely https://doi.org/10.1017/jfm.2014.13. This has been corrected in the original, a footnote added to explain the circumstances, and the record in CrossRef updated.

We apologise to the authors of the two articles, and to readers who have been affected in their navigation to the respective articles.

\section{REFERENCES}

Guardone, A., Colonna, P., Casati, E. \& Rinaldi, E. 2014 Non-classical gas dynamics of vapour mixtures. J. Fluid Mech. 741, 681-701. https://doi.org/10.1017/jfm.2013.13.

Weinstock, J. 2013 Analytical theory of homogeneous mean shear turbulence. J. Fluid Mech. 727, 256-281. https://doi.org/10.1017/jfm.2014.13. 